\title{
Effects of Ginkgo biloba on Diseases Related to Oxidative Stress
}

\author{
Authors \\ Gabriela Achete de Souza ${ }^{1}$, Sâmylla Vaz de Marqui ${ }^{1}$, Júlia Novaes Matias ${ }^{1}$, Elen Landgraf Guiguer ${ }^{1,2}$,
} Sandra Maria Barbalho ${ }^{1,2}$

Affiliations

1 Department of Biochemistry and Pharmacology, School of Medicine, University of Marília (UNIMAR), Marília, São Paulo, Brazil

2 Department of Biochemistry and Nutrition, Faculty of Food Technology of Marília, (FATEC), Marília, São Paulo, Brazil

\section{Key words}

Ginkgo biloba, Ginkgoceae, antioxidant, oxidative stress, anti-inflammatory

\author{
received December 15, 2019 \\ revised January 21,2020 \\ accepted January 29, 2020
}

\author{
Bibliography \\ DOI https://doi.org/10.1055/a-1109-3405 \\ published online February 25, 2020 | Planta Med 2020; 86: \\ 376-386 @ Georg Thieme Verlag KG Stuttgart · New York | \\ ISSN 0032-0943 \\ Correspondence \\ Dr Sandra Maria Barbalho \\ Department of Biochemistry and Pharmacology, \\ School of Medicine, University of Marília \\ Av. Higino Muzzi Filho 1001, Marília 17525-902, SP, Brazil \\ Phone: + 5514996553190 , Fax: + 551421054000 \\ smbarbalho@gmail.com
}

\section{ABSTRACT}

Ginkgo biloba (GB) is one of the most widely used phytotherapeutic products in the world, and its extract has beneficial properties for the treatment of several pathologies, such as diabetic cardiomyopathy, neurodegenerative diseases, cataracts, hearing loss, myocardial lesion, hippocampus neuronal lesions, morphometry testicular changes, and liver damage. This review aims to investigate the effects of GB on diseases related to oxidative stress. Databases such as MEDLINE/ PUBMED and EMBASE were consulted, and PRISMA guidelines were used to build the review. This plant has antioxidant properties since it regulates the expression of antioxidant enzymes positively and reduces reactive oxygen and nitrogen species, contributing to the reduction of lipid peroxidation. It also exhibits anti-inflammatory properties, inhibiting the expression of pro-inflammatory cytokines, such as IL-1, IL-6, and TNF- $\alpha$. In animal models, the use of GB can show positive effects on brain damage, neurodegenerative diseases, myocardial injury, and renal and liver damage. In humans, the positive effects were shown in diabetes, metabolic syndrome, and ischemic colitis. These effects are due to the presence of compounds such as bilobalide, isoramnetina, quercetin, kaempferol, and ginkgolides A, B, and C. For these reasons, GB can be a lowcost alternative to the therapeutic approach of several pathologies since it acts in the prevention, treatment, and inhibition of several complications of common comorbidities.

\section{Introduction}

Oxidative stress is caused by destructive and progressive modifications in one or more body tissues, leading to dysfunction of organs, premature aging, and sometimes disease and death. It is a natural and fundamental process of the body, but it also involves the acceleration of destructive modifications over time, not only at the cellular level but also at the molecular level [1,2].

On an ongoing basis, the cells produce oxygen (ROS) and nitrogen (RNS) reactive species as part of metabolic processes during stress exposure, radiation, infections, and smoke exposure. These substances cause significant damage in organic biomolecules (nucleic acids, lipids, and proteins), inducing alterations in DNA, which, in turn, affect homeostasis and can lead to several oxidative disorders related to stress, such as cardiovascular diseases and cancer. It is possible that the usage of phytotherapeutic products, such as Ginkgo biloba (GB), may delay this process $[3,4]$.
GB belongs to the Ginkgoceae family, and it is one of the oldest living species on the planet. The leaves and seeds of this plant have been used for medicinal purposes in China for centuries, initially being used for asthma and problems in the digestive system. In Europe and the USA, they have been sold since the sixties, and now they represent one of the most popular phytotherapeutic products in the world [5-12].

Therefore, this study aimed to review the effects of GB on oxidative processes.

\section{Methodology}

\section{Data source}

To carry out this review, we used the MEDLINE-PubMed (National Library of Medicine, National Institutes of Health) and EMBASE databases to retrieve studies from May 2013 to October 2019), fol- 
lowing the PRISMA guidelines (Preferred Reporting Items for Systematic Reviews and Meta-Analyses). This review was conducted to answer the following question: What are the effects of $G B$ on diseases related to oxidative stress?

\section{Research}

This research includes placebo-controlled randomized clinical trials, case-control retrospective studies, and prospective transversal studies. Experimental model studies were also conducted. The combinations of used terms for this research were Ginkgo biloba and oxidative stress, and Ginkgo biloba and inflammation. These combination of terms resulted in a list from which we selected 58 articles that were used to build $\downarrow$ Table 1 . The flowchart shows the selection of articles, as well as the inclusion and exclusion of studies ( $>$ Fig. 1). Other studies about GB, inflammation, oxidative stress, and antioxidants were used in the discussion section.

\section{Eligible criteria and study selection}

This research includes quantitative and qualitative studies that reported the usage of GB in the treatment of diseases caused by oxidative stress and inflammation. All the articles relating to $G B$ and oxidative stress and $G B$ and inflammation published in the last $5 \mathrm{y}$ were included, except for revisions, communication letters, and non-English articles.

\section{Data extraction}

Data extraction was performed by 3 authors who used the pre-defined data above. The data was extracted from articles that included the date, author, specimen size, gender, diseases related to oxidative stress and inflammation, and the usage of GB extract (EGB761). Disagreements between the above reviewers were evaluated and resolved by a third reviewer.

Only original articles were selected. The exclusion criteria for this research were revisions, non-English studies, case reports, not full-text articles, editorials, and poster presentations. Revisions were used to help in the discussion but were not included in $>$ Table 1.

\section{Results}

In the last 5 y, 54 studies showed the potential of EGB761 and its components in animal models. Most of them were performed on mice of varying species (Wistar, Sprague-Dawley, C57BL, Balb/c, ICR, hairless), and remaining studies were conducted on humans. All the research studies were developed in universities and health care centers, and the articles are summarized in $>$ Table 1 .

\section{Discussion}

The increase in chronic degenerative diseases has led to a growing interest in phytotherapeutic products since they are low-cost options and normally do not have important adverse effects. In that context, EGB761 presents an essential relevance for its antioxidant and anti-inflammatory properties [9-11].

EGB761 has been used for the treatment of a series of comorbidities related to oxidative stress and, due to its antioxidant na- ture, it can decrease oxidative processes and neutralize lipid peroxidation. Among the bioactive components of EGB761, we can highlight flavonoids (about 28\%) such as quercetin, kaempferol isorhamnetin, lipoxygenase, phospholipase $A 2$, and terpenic lactones (2.8-3.4\% of ginkgolídeos A, B, and C and 2.6-3.2\% bilobalide) ( $\bullet$ Table 2) $[9,13,14]$.

\section{$\mathrm{GB}$ and inflammation}

A lesser known property of EGB761 is its anti-inflammatory action both in human and animal models and in vitro studies. Currently, it is known that its components, mainly ginkgolide $A$, can suppress cyclo-oxygenase-2 (COX-2) and 5-lipo-oxygenase (5-LOX), which are limiting enzymes for the conversion of arachidonic acid to prostaglandin and leukotrienes particularly, and it also can reduce endoplasmic reticulum stress, which would be responsible for boosting the inflammation. It was found that EGB761 can inhibit the effects of the lipopolysaccharide (LPS) by improving the action of transforming growth factor (TGF), thus reducing the gene expression of Interleukin-1 (IL-1), IL-6, and tumor necrosis factor alpha (TNF- $\alpha$ ) ( $\triangleright$ Fig. 1), resulting in down-regulation of the inflammatory processes. It is also known that ginkgolide B can inhibit platelet-activating factor (PAF), which plays a large part in the inflammation of the airways $[12,13,15-20]$. Although the reduction of pro-inflammatory cytokines has been identified in the studies, little is known about the mechanism of this reduction. The most known mechanism is illustrated in $\mathbf{F i g} .2$.

EGB761 is very well-known for its neuroprotective activity and for promoting memory improvement. Both factors are directly related to its antioxidant properties. The components of EGB761 perform several actions in the regulation of oxidative stress, and among these actions, the main mechanisms are the capture of free radicals and the indirect inhibition of free radicals formation [13].

Each of the components shows specific actions on proteins and specific metabolic pathways, thus contributing to oxidative stress attenuation, according to $\mathbf{\sim}$ Table 2 .

Besides the specific actions of each component, other advantages have been attributed to EGB761. As an example, its usage in neurology can improve circulation because it can reduce the peroxide level in cerebellar neurons and protect the cortical neurons from iron-induced injuries. It also reduces ROS and RNS, hydroxyl radicals $(\mathrm{OH})$, peroxyl radicals (ROO), anion superoxide radical (O2-), nitric oxide, and hydrogen peroxide radical $\left(\mathrm{H}_{2} \mathrm{O}_{2}\right)$. It positively regulates the expression of RNAm of antioxidant enzymes, such as mitochondrial superoxide dismutase (MnSOD) and glutathione peroxidase (GPx) [10, 13, 21,22]. $>$ Fig. 3 presents the effects of $G B$ on inflammation and oxidative stress.

\section{GB and oxidative stress}

Many diseases are related to oxidative stress, such as hearing loss, where there is observed a critical increase of ROS in the cochlea blood flow; this same excess in the eyes may lead to cataracts. Oxidative stress leads to lipid peroxidation, protein oxidation, and DNA mutation, causing damage to nerve cells and being responsible for neurological disorders [23-27].

Besides being related to the origin of diseases, oxidative stress also has an impact on the aggravation of other pathologies, as in 

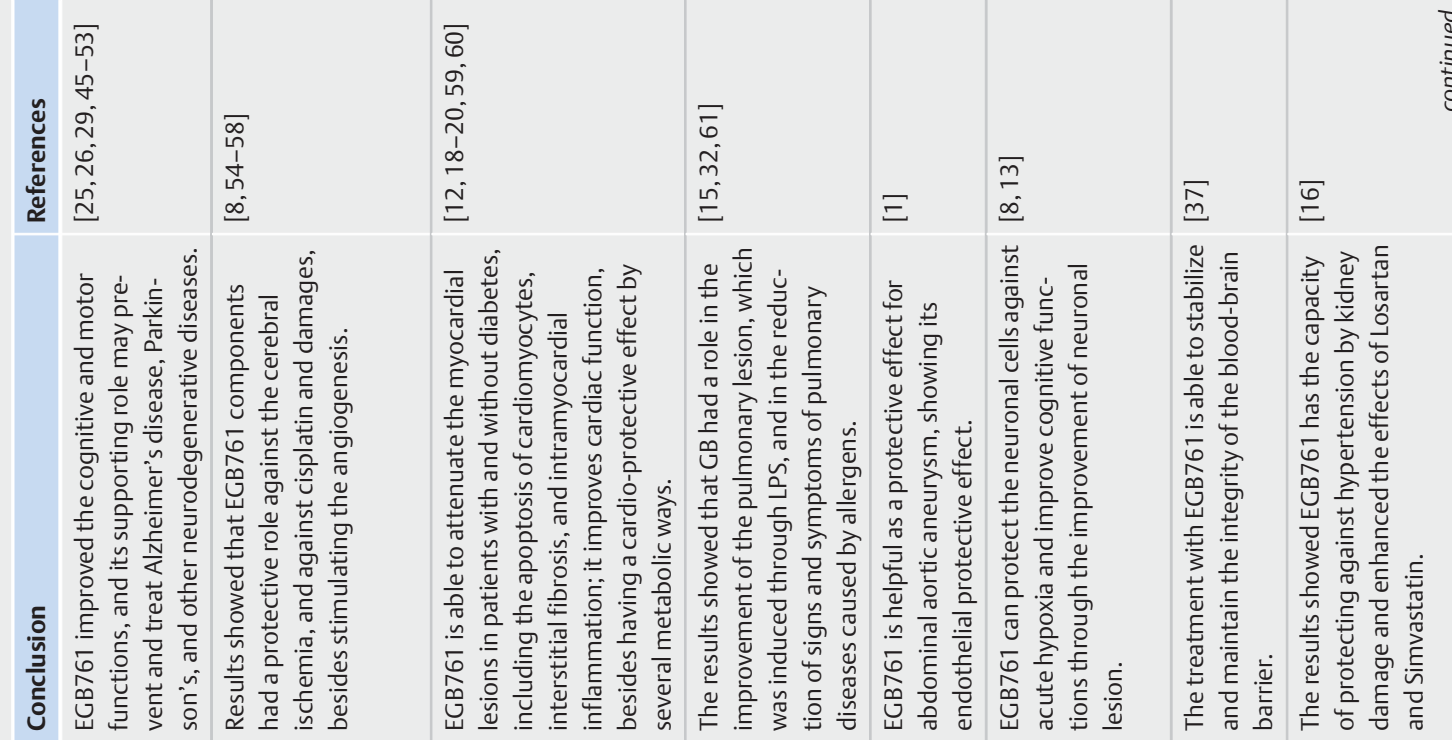

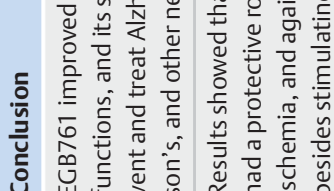

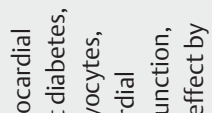

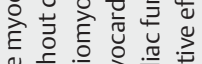

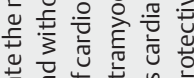

苋语

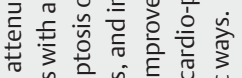

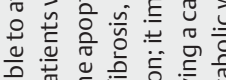

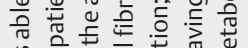

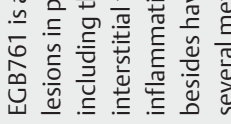

突

亭 $\frac{\bar{E}}{\bar{U}}$

กิ

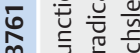

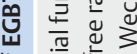

는 虫

은 흐

艺

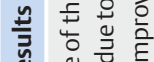

呓.

焉

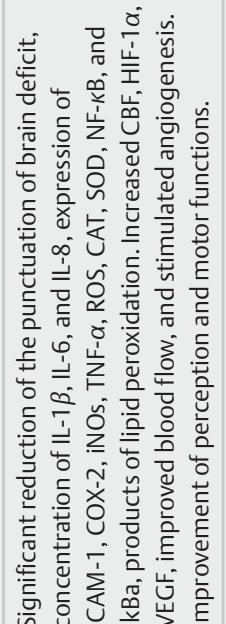

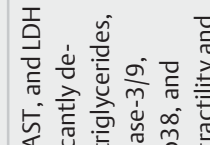

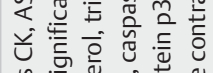

๗

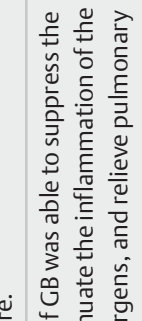

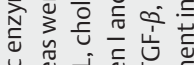

t.

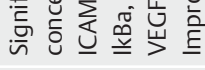

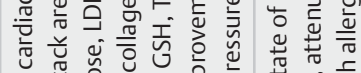

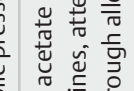

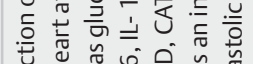

त站

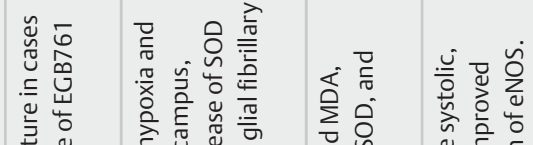

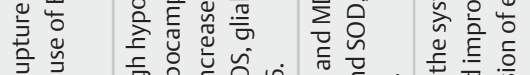

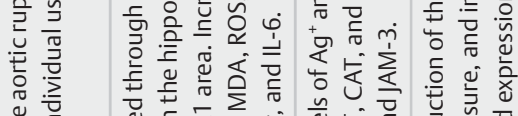

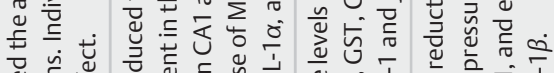

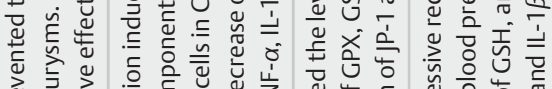

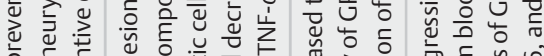

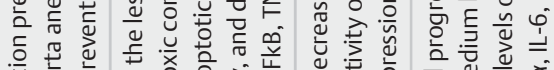

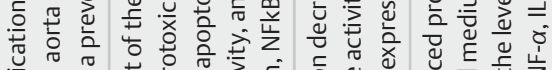

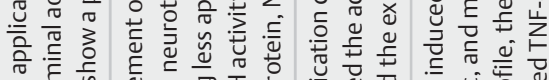

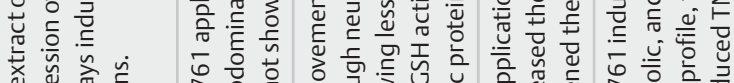

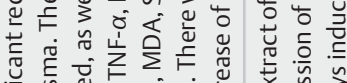

站

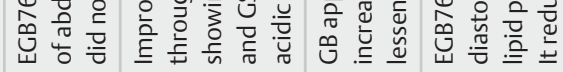

它 它

ㄷㅇㅇ응

证

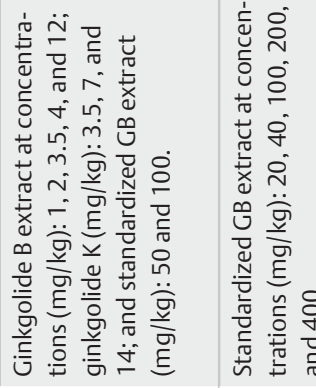

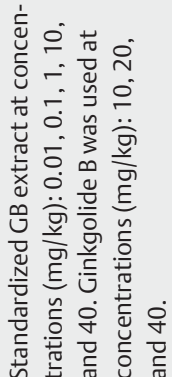

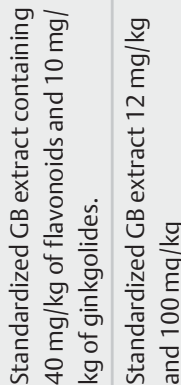

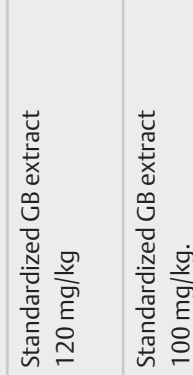

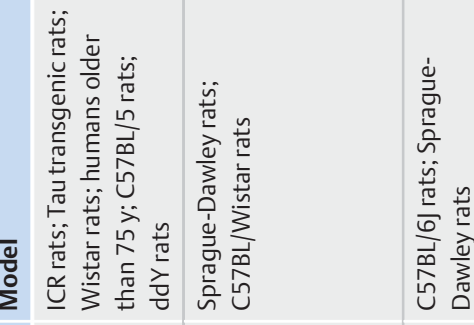

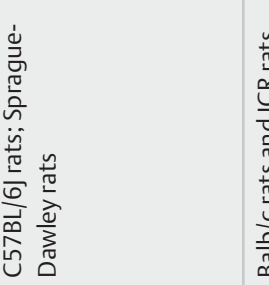

0

गे

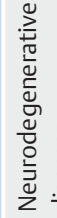

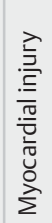

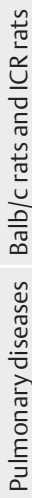

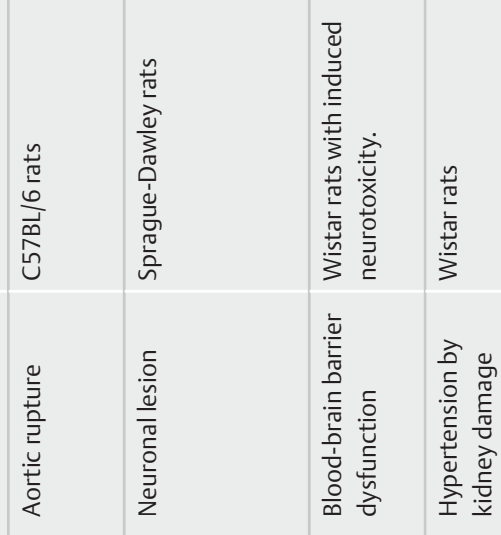




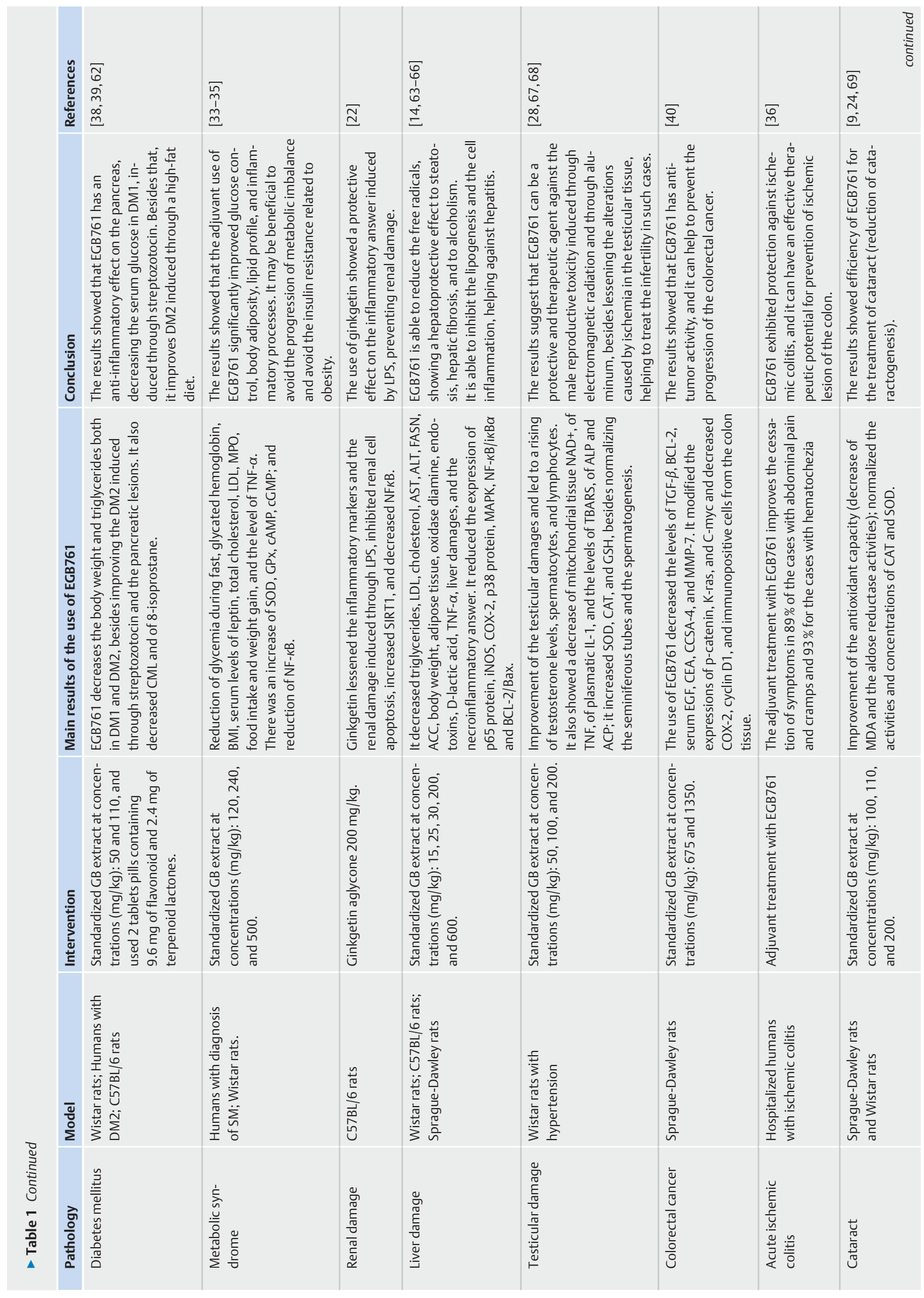




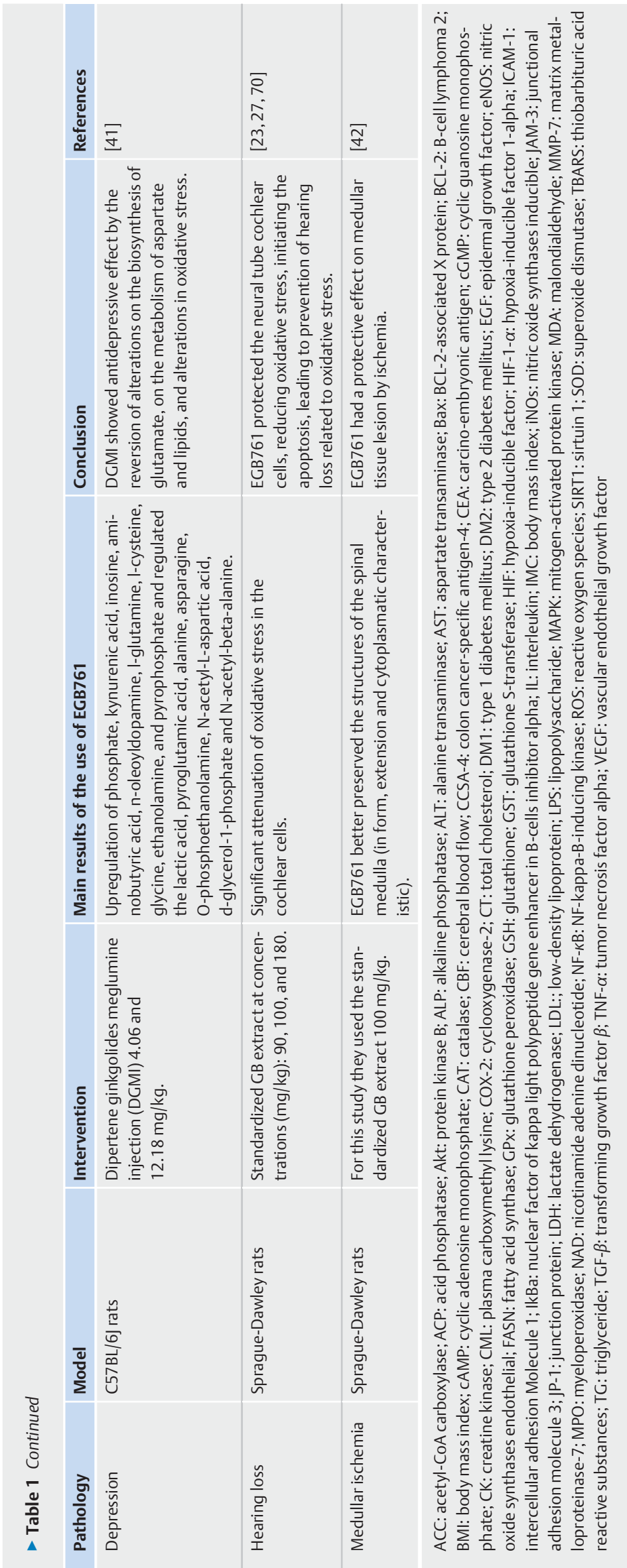




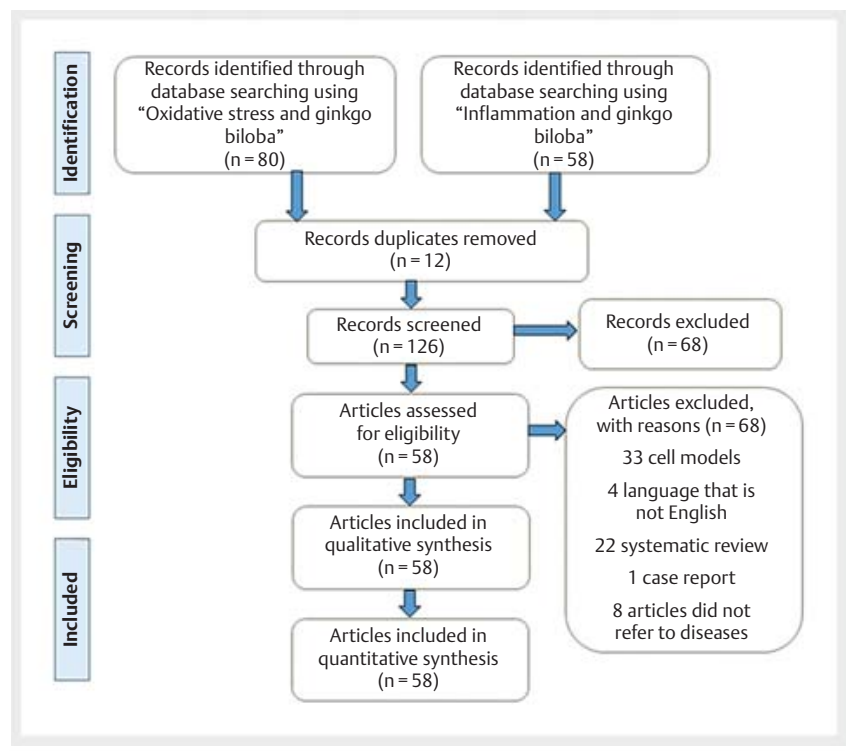

- Fig. 1 Flow diagram for the literature search (based on PRISMA, 2009).

the case of diabetes, in which hyperglycemia leads to oxidative stress, resulting in cardiomyopathy. The same happens after partial hepatectomy, in which the free radicals harm liver regeneration. In addition to those cases, oxidative stress can reduce the total number of moving sperm cells and induce lesions to the myocardium during the heart reperfusion [12,14,28-30]. In all these cases, it is possible to use EGB761 ( $\bullet$ Table 1 ).

To investigate the effects of EGB761 on cognitive functions, Belviranli and Okudan [25] performed research on Wistar mice and found out that, with the supplementation of EGB761, there was significant improvement not only in the locomotor activity but also in anxiety, spatial learning, and memories. Another important discovery of this research was that in older rats, there was an improvement of the oxidative damage in cerebral tissue. Beyond this research, there were 11 more experimental studies in which it is possible to realize the positive impact of EGB761 in degenerative diseases.

According to Tang et al. [12], the free radicals derived from oxygen are known for playing a critical role in the genesis of heart tissue injury. Under normal conditions, cells can suppress free radicals. However, the unbridled formation of free radicals promotes an imbalance. Pathological conditions, such as ischemic-reperfusion, may cause this imbalance. This study showed that the administration of EGB761 in mice inhibited the signaling pathways of toll-like receptor- 4 and nuclear factor- $\kappa \beta$ (NF- $\kappa \beta)$, showing protective effects against the ischemic-reperfusion lesion because of its anti-inflammatory effects.

According to Tao et al. [15], the administration of EGB761 showed significant decreases in the release of IL-4, IL-5, IL-6, IL-8, IL-13, and TNF- $\alpha$ in allergic mice. EGB761 was also responsible for adjusting the leukocyte elastase, an active protein in blood coagulation disorders, chronic bronchitis, and pulmonary injury. The components of EGB761 were able to reduce airways inflammation. Wu et al. [31] showed that ginkgolides play a vital role in the alleviation of LPS-induced pulmonary injury. Similarly, Lee et al. [32] concluded that the expression of pro-inflammatory mediators, such as TNF- $\alpha$, IL-6, macrophage inflammatory protein (MIP)-2, nitric oxide synthase (NOS) and COX-2, was suppressed in adult mice. EGB761 also reduced the activation of NF-kappa-Binducing kinase (NFKB) and the phosphorylation of NF-kappa-B inhibitor $\left(I_{\kappa} \mathrm{B}\right)$.

The study performed by Aziz et al. [33] evaluated the effects of EGB761 (120 mg a day) in patients taking Metformin for 90 days, comparing them to a placebo group. It was possible to conclude that the administration of EGB761 decreased the levels of HbA1c, glycemia, insulin, insulin resistance, abdominal circumference, serum leptin, and inflammatory markers. It did not cause liver, renal, and hematopoietic system damage but showed significant improvement in patients with metabolic syndrome. Regarding metabolic syndrome, Hirata et al. [34] showed that the administration of EGB761 in obese mice $(500 \mathrm{mg} / \mathrm{kg}$ ) promoted a significant decrease in feed intake and decreased weight in comparison controls, showing that EGB761 is an option for treatment of obese patients who are resistant to nutritional education treatment. The work of Siegel et al. [35], even with a small number of participants, showed the capacity of EGB761 in diminishing the homeostatic model assessment of insulin resistance (HOMA-IR), the C-reactive protein (CRP), and IL-6, concluding that EGB761 can be used as a complementary drug with preventive potential for cardiovascular diseases, a common cause of death in patients with metabolic syndrome.

EGB761 presents an option for the treatment of acute ischemic colitis patients, according to Fang et al. [36]. The study was performed by selecting acute ischemic colitis patients, who were separated into 2 groups: the EGB761 group $(n=30)$ and the routine group $(n=17)$. The members of the EGB761 group received a routine of intravenous injections with EGB761. EGB761 decreased abdominal pain and hematochezia and promoted a significant attenuation of macroscopic and histological damages in the patient's colon. There was an increase in the levels of superoxide dismutase (SOD) and a decrease in the levels of malondialdehyde (MDA), TNF- $\alpha$, and IL-6. However, the number of patients in this study was small. Thus, it would also be viable if further research were done in order to enable this treatment in a noninvasive way, because the administration of intravenous injections might impede the compliance of patients with limited access to health services to receive the treatment.

An abdominal aortic aneurysm is a vascular disease characterized by a luminal pathological dilatation, and one of its fatal consequences is the aortic rupture. To evaluate the therapeutic effect of EGB761 on the aortic rupture, Huang et al. [1] administered angiotensin II (Ang II) in mice for 28 days, causing abdominal aortic aneurysm, and then administered EGB761 (100 mg/kg/day) for 56 days. It was possible to conclude that EGB761 prevents an aortic rupture in hypercholesterolemic mice, which were infused with Ang II, but only in the initial stage, restricting the protective range of this treatment in the mentioned comorbidity.

According to Ekici, Muhtaroğlu and Bedirli [14], after partial hepatectomy and liver transplant, the liver regeneration is awaited, for it is a vital process. After hepatectomy, the free radicals that are released by the peroxidation cause damage to tis- 
- Table 2 Components of Ginkgo biloba and their respective actions related to oxidative stress.

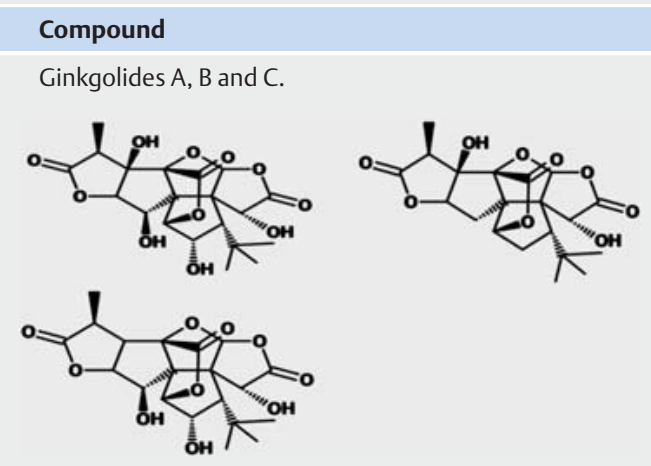

Kaempferol

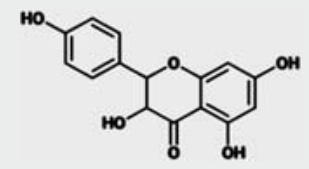

Quercetin

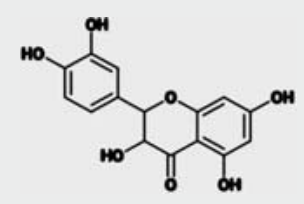

Bilobalide

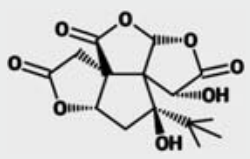

Isorhamnetin

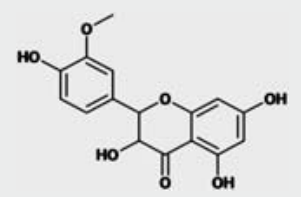

\section{Action}

Decrease the level of ROS, the release of LDH, TNF- $\alpha$, IL- $1 \beta$ and IL- 6 and the expression of $\mathrm{c}$-fos and $\mathrm{c}$-jun mRNA; inhibits activation factor of platelets and of signaling pathway NIK/IKK $\alpha / \mathrm{IkB} / \mathrm{NF}-\kappa \mathrm{B}$; increases cellular proliferation, activity of free radicals capture, activation of the p42/p44 (ERK) MAPK pathways, levels of mRNA and the protein from the HIF- $1 \alpha$.
References

[71-74]

$[43,74,75]$

Increase of the expression of GCLC, BDNF, BCL-2, and GSH; inhibits serotonin degradation by $\mathrm{MAO}$, liberation of the Cytochrome $\mathrm{C}$, activity of Caspase-3, fragmentation of the internucleosome DNA, $\mathrm{NADH}$, activation of $\mathrm{p} 53$ protein, $\mathrm{p} 65$ protein, NF- $\kappa \mathrm{B}$ and apoptosis; decrease in the neurotoxicity induced through 3-NP and the elevation of BAX through ROS; elimination of free radicals; and upregulation of HMOX-1.

Increase the level of BDNF; inhibition of degradation of serotonin by $\mathrm{MAO}$, apoptosis; transcription of TNF- $\alpha$; activation of ERK, phosphorylation, and activation of JNK; decrease of lipid peroxidation in the plasma and phosphorylation of $\mathrm{lkB} \beta$; upregulation of HMOX-1; and elimination of free radicals.
Decrease in the expression of reactive species induced through $\mathrm{H}_{2} \mathrm{O}_{2}$; the elevation of $\mathrm{BAX}$ induced through ROS, the c-myc and p53proteins; upregulation of $\mathrm{BCL}-2$, of the sub-unit III from the cytochrome c oxidase, of the sub-unit ND1 from the NADH dehydrogenase, and of the CREB-BDNF pathway. Inhibition of the degradation of membrane phospholipids; activity of the caspase-3. Increase of cellular proliferation of neurons of the hippocampus.

Decrease of cellular death and fragmentation of DNA. Inhibition of apoptosis, liberation of cytokines from the mitochondria, and cleavage of PARP, the ERK pathway, and the activation of $\mathrm{p} 53$ protein. Upregulation of genes related to $\mathrm{BCL}-2$; downregulation of $\mathrm{BH} 3$ gene and genes related to BAX. Elimination of ROS.

3-NP: 3-nitropropionic acid; BAX: BCL-2 associated protein X; BCL-2: B-cell lymphoma protein 2; BDNF: brain-derived neurotrophic factor; BDNF: brainderived neurotrophic factor; $c$-fos: gene c-fos; c-jun: gene c-jun; CREB: CAMP response element-binding protein; ERK: extracellular signal-regulated kinases; GCLC: glutamate-cysteine ligase catalytic subunit; GSH: glutathione; HIF-1 $\alpha$ : hypoxia-inducible factor 1-alpha; HMOX-1: Heme oxygenase 1; IkB: NF-kappa-B inhibitor; IkB- $\beta$ : NF-kappa-B inhibitor beta; IKK $\alpha$ : IKB kinase $\alpha$; IL: interleukin; JNK: c-Jun N-terminal kinase; LDH: lactate dehydrogenase; MAO: monoamine oxidase; MAPK: mitogen-activated protein kinase; mRNA: RNA messenger; NADH: dinucleotide of de nicotinamide and adenine; NF- $\mathrm{KB}$ : NF-kappa-B-inducing kinase; NIK: NF-kappa-B-inducing kinase; PARP: poly adenosinediphosphate ribose; ROS: reactive oxygen species; TNF- $\alpha$ : alpha tumoral necrosis factor

sues, and inactivation of the free radicals makes possible anti-inflammatory effects and speeds up the regeneration process. This study showed positive results with the intraperitoneal administration of EGB761 due to antioxidant and anti-inflammatory effects, as well as raising in the mitotic index.

According to Kaur, Sharma, and Nehru [13], neurotoxic disorders are one of the significant causes of death and tissue lesions. The trimethyl-tin (TMT), known for being a powerful neurotoxic product, was used to evaluate the protective effects against neuronal damage in the hippocampus of mice administrated with
EGB761. This study showed a significant cognitive improvement, as well as a decrease of free radicals and pro-inflammatory cytokines, demonstrating that EGB761 is an effective agent against neuronal damage of the hippocampus inducted through TMT.

To evaluate the capacity of EGB761 to maintain the integrity of the hematoencephalic barrier, Lebda et al. [37], administrated $50 \mathrm{mg} / \mathrm{g}$ of AgNPs (silver nanoparticles) in mice by intraperitoneal route, inducing neurotoxicity. After the administration of EGB761 $(120 \mathrm{mg} / \mathrm{kg})$, the authors observed that EGB761 was able to neutralize the adverse effects of AgNPs, stabilizing the integrity of the 


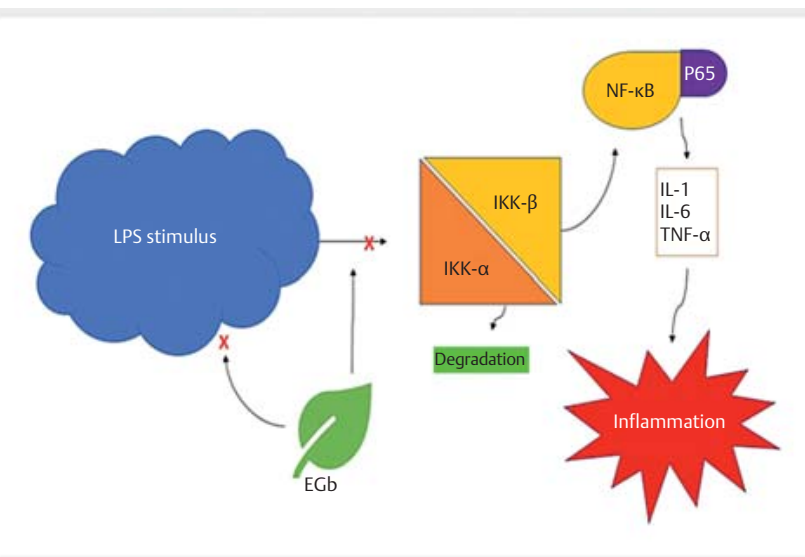

- Fig. 2 EGB761 acts on the LPS, which unleashes events of transduction of serial signal including activation of NF- $\mathrm{kB}$, which regulates the production of pro-inflammatory cytokines. LPS: lipopolysaccharide; EGB761: Ginkgo biloba extract; IKK- $\beta$ : inhibiting nuclear factor kappa-B kinase subunit $\beta$; IKK- $\alpha$ : ihibiting nuclear factor kappa-B kinase subunit kinase subunit $\alpha$; NF-KB: nuclear factor kappa-B; P65: transcription fFactor p65; IL: interleukin; TNF- $\alpha$ : tumoral necrosis factor $\alpha$.

hematoencephalic barrier by its anti-inflammatory, antioxidant, and anti-apoptotic properties, and by the improvement of protein of the tight junction.

Several studies have been done to analyze the action of EGB761 in the treatment of type 2 diabetes mellitus (DM2). According to the study of Zayed et al. [38], the administration of EGB761 in mice with inducted DM2 resulted in the reduction of glycemia in a very significant way and reduced the levels of urea and creatinine in comparison to diabetic mice that were not treated. The work of Rhee et al. [39] induced type 1 diabetes mellitus (DM1) and DM2 in mice and showed that the use of EGB761 reduced glycemia and levels of insulin. They also showed higher levels of hepatic lipoprotein lipase (LPL) and peroxisome proliferatoractivated receptor alpha (PPAR- $\alpha$ ), while Interleukin 1-beta (IL-1 $\beta$ ) and the TNF- $\alpha$ were reduced. In the model in which DM2 was simulated, the mice that received EGB761 showed lower levels of triglycerides, higher levels of hepatic LPL and PPAR- $\alpha$, and a decrease in body weight. Therefore, it is possible to conclude that EGB761 can have protective effects on patients with DM1 and DM2. However, there is still a lack of study models in human beings to corroborate such effects, which once proved, can be used by the population to prevent DM complications.

According to Gevrek et al. [28], radiation produced by electromagnetic mobile phones can lead to oxidative stress, being responsible for causing testicular morphometry damages, decreasing the number of moving spermatozoids, and decreasing testosterone levels. This study showed beneficial results after the administration of EGB761 in mice that were subjected to radiationreducing the toxic effects, reverting the damage to the testicular tissue, and restoring spermatogenesis and hormonal levels-suggesting that EGB761 is a protective and therapeutic agent against male reproductive toxicity induced by radiation.

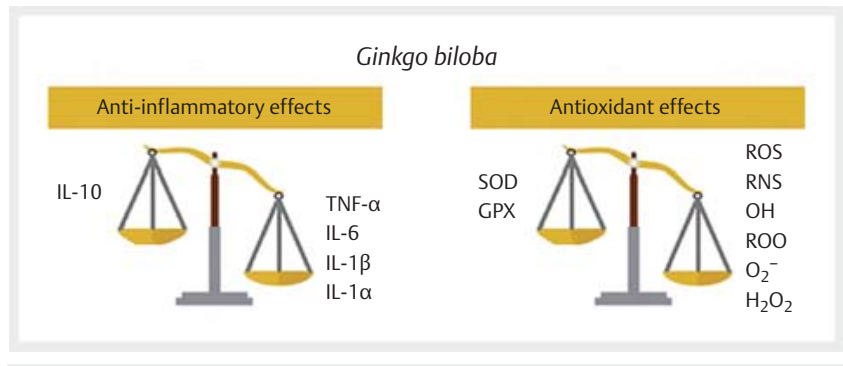

- Fig. 3 Effects of Ginkgo biloba on inflammatory processes and oxidative stress. IL-10: interleukin 10; TNF- $\alpha$ : tumoral necrosis factor $\alpha$; IL-6: interleukin 6; IL-1 $\beta$ : interleukin 1-beta; IL-1 $\alpha$ : Interleukin 1-alpha; SOD mitochondrial: superoxide dismutase; GPx: glutathione peroxidase; ROS: oxygen reactive species; RNS: nitrogen reactive species; $\mathrm{OH}$ : hydroxyl radicals; $\mathrm{ROO}$ : peroxyl radicals; $\mathrm{O}_{2}{ }^{-}$: Superoxide Anionic Radical; $\mathrm{H}_{2} \mathrm{O}_{2}$ : hydrogen peroxide.

In the study by Abdel-Zaher et al. [16] hypercholesterolemia hypertension in mice was induced through methyl ester, nitro arginine, and food with 1\% cholesterol. EGB761 was able to decrease hypertension caused by liver damage induced through hypercholesterolemia, reducing the expression of eNOS (endothelial nitric oxide synthase) and raising the expression of iNOS (inducible nitric oxide synthase), TNF- $\alpha$, IL-6, and IL- $1 \beta$.

The use of EGB761 for the treatment of colorectal cancer was evaluated during the study of Ahmed et al. [40]. Colorectal cancer was induced in mice through the administration of $\mathrm{N}$-methyl nitrosourea by rectal route, 3 times a wk, for $5 \mathrm{wks}$, followed by treatment with EGB761 in a dose of $1.35 \mathrm{~g} / \mathrm{Kg}$. It was observed that the mice that received EGB761 had suppression of the proliferation of tumor cells, as well as a promotion of apoptosis. The study highlighted that the improvement of this condition may be attributed to the inhibition of the signaling module Wnt/ $\beta$-Catenin. The results suggest that EGB761 is an alternative treatment approach to this type of cancer.

To analyze the effectiveness of GB for the treatment of kidney damage, Zhang et al. [22] used ginkgetin aglycone (GA), a new EGB761 with higher liposolubility and a higher antioxidant effect. The administration of GA protected against acute kidney damage induced through LPS and helped in relieving the inflammatory process, the kidney damage, and the tubular apoptosis. The GA avoided the kidney damage and activated Sirtuin 1 (SIRT1) by the inactivation of the signaling route of NFkB.

In another study, Cao et al. [24] divided the mice into a control group, group II-mice that received selenite (nontreated)-and group III-mice that received selenite + EGB761. The groups II and III received $19 \mathrm{mmol} / \mathrm{kg}$ of selenite, and group III received, in addition to it, $0.35 \% 100 \mathrm{mg} / \mathrm{kg}$ of EGB761 Selenite, which was used to decrease cataractogenesis. When examining both eyes of the mice, it was possible to notice that $83 \%$ of the mice of group II showed dense opacification of the lens (grade +++ ), while in group III, only $25 \%$ showed a light opacification (grade + ). All mice in the control group showed full transparency of the lens (grade 0 ). These results show that the use of EGB761 can decrease the cataractogenesis process. 
The effect of EGB761 on the treatment of depression was studied by Liang et al. [41]. The study analyzed the effects of diterpene ginkgolide (DG), an essential class of EGB761 components. The results showed that there were significant alterations in metabolism levels of neurotransmitters, oxidative stress, glutathione (GSH) metabolism, lipid metabolism, energetic metabolism, and kynurenic acid in the hippocampus. DG can exert an antidepressive role by the reversion of the alterations in the biosynthesis of glutamate, aspartate metabolism, oxidative stress, neural inflammation, and lipid and energy metabolism of the hippocampus. DG also raised the levels of gamma-aminobutyric acid (GABA), inosine, and kynurenic acid. As the levels of depression have been exponentially rising all around the world, it is of utmost importance that further research on this topic is done aiming at a more diverse range of treatments for this comorbidity.

Wang and Wang [23] investigated the use of EGB761 for the protection of cochlear neural stem cells (NSCs) and showed that this compound raises the cell viability, decreasing the oxidative stress-induced through $\mathrm{O}_{2}$, beyond preventing mitochondrial depolarization and, as a consequence, apoptosis.

Park et al. [27] carried out a study to investigate the effects of renexin (a drug made of the combination of Cilostazol and EGB761) on the injury caused to the Corti organ by noise and to the medial olivocochlear system of mice, and showed that the treatment may lead to the recovery of the injuries caused by the noise.

The work of Badem et al. [42] evaluated the effect of EGB761 in medullar ischemia. For that, researched clamped the infrarenal abdominal aorta in mice, which were divided into groups, and values of MDA, SOD, GSH, and GPx tissue, as well as tissue from spinal cord samples, were analyzed. The mice that received the EGB761 showed a smaller medullar lesion in comparison to other groups. However, it was not possible to demonstrate a uniform effect of the action of EGB761 on the biochemical markers of ischemia/reperfusion lesion.

In all the studies of this review, it was possible to verify that no significant adverse effects are observed after the use of EGB761. However, research carried out by Jiang et al. [11] showed that a high level of ginkgolic acid could be hepatotoxic.

Besides the diseases related above, other comorbidities show an improvement with the use of EGB761, such as psychiatric disorders, vitiligo, chronic alcoholic myopathy, glaucoma, and renal hypertension $[16,43,44]$.

To the best of our knowledge, this is the first review showing the effects of GB on diseases related to oxidative stress.

\section{Conclusion}

$G B$, as well as its extract, has beneficial properties for the promotion and management of health because of its wide-ranging antiinflammatory and antioxidant properties. Considering that diseases that are caused by oxidative stress have reached epidemic proportions worldwide, it is necessary to find accessible and effective alternatives that may minimize the risk factors for these diseases and contribute to the treatment.

More studies are necessary to enlighten the GB properties and applications in the pharmaceutical industry, as well as more infor- mation about doses, the best administration route, and pharmaceutical formulation in order to provide validation for its medicinal use.

\section{Conflict of Interest}

The authors declare that they have no conflict of interest.

\section{References}

[1] Huang XF, Zhang SZ, You YY, Zhang N, Lu H, Daugherty A, Xie XJ. Ginkgo biloba extracts prevent aortic rupture in angiotensin II-infused hypercholesterolemic mice. Acta Pharmacol Sin 2019; 40: 192-198

[2] Burgos-Moron E, Abad-jimenez Z, Maranon AM, lannantuoni F, Escribano-Lopez I, Lopez-Domenech S, Salom C, Jover A, Mora V, Roldan I, Sola E, Rocha M, Victor V. Relationship between oxidative stress, ER stress, and inflammation in type 2 diabetes: the battle continues. J Clin Med 2019; 8: 1385

[3] Senoner T, Dichtl W. Oxidative stress in cardiovascular diseases: still a therapeutic target? Nutrients 2019; 11: 2090

[4] Bjorklund G, Dadar M, Martins N, Chirumbolo S, Goh BH, Smetanina K, Lysiuk R. Brief challenges on medicinal plants: an eye-opening look at ageing-related disorders. Basic Clin Pharmacol Toxicol 2018; 122: 539558

[5] Hirata BKS, Pedroso AP, Machado MMF, Neto NIP, Perestrelo BO, de Sa R, Alonso-Vale MIC, Nogueira FN, Oyama LM, Ribeiro EB, Tashima AK, Telles MM. Ginkgo biloba extract modulates the retroperitoneal fat depot proteome and reduces oxidative stress in diet-induced obese rats. Front Pharmacol 2019; 10: 686

[6] Li W, Qinghai S, Kai L, Xue M, Lili N, Jihua R, Zhengxiang L, Xiaoling L, Di G, Qi Y, Mengyun D, Jianfeng F. Oral administration of ginkgolide B alleviates hypoxia-induced neuronal damage in rat hippocampus by inhibiting oxidative stress and apoptosis. Iran J Basic Med Sci 2019; 22: 140145

[7] Martinez-Solis I, Acero N, Bosch-Morell F, Castillo E, Gonzalez-Rosende ME, Munoz-Mingarro D, Ortega T, Sanahuja MA, Villagrasa V. Neuroprotective potential of Ginkgo biloba in retinal diseases. Planta Med 2019; 85: $1292-1303$

[8] Li H, Sun X, Yu F, Xu L, Miu J, Xiao P. In silico investigation of the pharmacological mechanisms of beneficial effects of Ginkgo biloba I. on Alzheimer's disease. Nutrients 2018; 10: e589

[9] Khedr MH, Shafaa MW, Abdel-Ghaffar A, Saleh A. Radioprotective efficacy of Ginkgo biloba and Angelica archangelica extract against technetium-99 m-sestamibi induced oxidative stress and lens injury in rats. Int J Radiat Biol 2018; 94: 37-44

[10] Wang H, Wu X, Lezmi S, Li Q, Helferich WG, Xu Y, Chen H. Extract of Ginkgo biloba exacerbates liver metastasis in a mouse colon cancer Xenograft model. BMC Complement Altern Med 2017; 17: 516

[11] Jiang L, Si ZH, Li MH, Zhao H, Fu YH, Xing YX, Hong W, Ruan LY, Li PM, Wang JS. (1)H NMR-based metabolomics study of liver damage induced by ginkgolic acid (15:1) in mice. J Pharm Biomed Anal 2017; 136: 44-54

[12] Tang Y, Zhou G, Yao L, Xue P, Yu D, Xu R, Shi W, Yao X, Yan Z, Duan JA. Protective effect of Ginkgo biloba leaves extract, EGb761, on myocardium injury in ischemia reperfusion rats via regulation of TLR-4/NF-kappaB signaling pathway. Oncotarget 2017; 8: 86671-86680

[13] Kaur S, Sharma N, Nehru B. Anti-inflammatory effects of Ginkgo bilobo extract against trimethyltin-induced hippocampal neuronal injury. Inflammopharmacol 2018; 26: 87-104

[14] Ekici Gunay N, Muhtaroglu S, Bedirli A. Administration of Ginkgo biloba extract (EGb761) alone and in combination with FK506 promotes liver regeneration in a rat model of partial hepatectomy. Balkan Med J 2018; 35: $174-180$ 
[15] Tao Z, Jin W, Ao M, Zhai S, Xu H, Yu L. Evaluation of the anti-inflammatory properties of the active constituents in Ginkgo biloba for the treatment of pulmonary diseases. Food Funct 2019; 10: 2209-2220

[16] Abdel-Zaher AO, Farghaly HSM, El-Refaiy AEM, Abd-Eldayem AM. Protective effect of the standardized leaf extract of Ginkgo biloba (EGb761) against hypertension-induced renal injury in rats. Clin Exp Hypertens 2018; 40: 703-714

[17] Hu H, Li Y, Xin Z, Zhanga X. Ginkgolide B exerts anti-inflammatory and chondroprotective activity in LPS-induced chondrocytes. Adv Clin Exp Med 2018; 27: 913-920

[18] Zhang L, Liu J, Geng T. Ginkgetin aglycone attenuates the apoptosis and inflammation response through nuclear factor-kB signaling pathway in ischemic-reperfusion injury. J Cell Biochem 2018. doi:10.1002/jcb. 28086

[19] Tian J, Liu Y, Liu Y, Chen K, Lyu S. Ginkgo biloba leaf extract protects against myocardial injury via attenuation of endoplasmic reticulum stress in streptozotocin-induced diabetic ApoE(-/-) mice. Oxid Med Cell Longev 2018; 2018: 2370617

[20] Li Y, Zhang Y, Wen M, Zhang J, Zhao X, Zhao Y, Deng J. Ginkgo biloba extract prevents acute myocardial infarction and suppresses the inflammation and apoptosisregulating p38 mitogenactivated protein kinases, nuclear factorkappaB and Bcell lymphoma 2 signaling pathways. Mol Med Rep 2017; 16: 3657-3663

[21] Wang A, Yang Q, Li Q, Wang X, Hao S, Wang J, Ren M. Ginkgo biloba I. extract reduces $\mathrm{H} 2 \mathrm{O} 2$-induced bone marrow mesenchymal stem cells cytotoxicity by regulating mitogen-activated protein kinase (MAPK) signaling pathways and oxidative stress. Med Sci Monit 2018; 24: 31593167

[22] Zhang J, Yang S, Chen F, Li H, Chen B. Ginkgetin aglycone ameliorates LPS-induced acute kidney injury by activating SIRT1 via inhibiting the NF-kappaB signaling pathway. Cell Biosci 2017; 7: 44

[23] Wang C, Wang B. Ginkgo biloba extract attenuates oxidative stress and apoptosis in mouse cochlear neural stem cells. Phytother Res 2016; 30 : 774-780

[24] Cao S, Gao M, Wang N, Liu N, Du G, Lu J. Prevention of selenite-induced cataratogenesis by Ginkgo biloba extract (EGb761) in Wistar rats. Cur Eye Res 2015; 40: 1028-1033

[25] Belviranli M, Okudan N. The effects of Ginkgo biloba extract on cognitive functions in aged female rats: the role of oxidative stress and brain-derived neurotrophic factor. Behav Brain Res 2015; 278: 453-461

[26] El-Ghazaly MA, Sadik NA, Rashed ER, Abd-El-Fattah AA. Neuroprotective effect of EGb761(R) and low-dose whole-body gamma-irradiation in a rat model of Parkinson's disease. Toxicol Ind Health 2015; 31: 11281143

[27] Park SY, Back SA, Kim HL, Kim DK, Yeo SW, Park SN. Renexin as a rescue regimen for noise-induced hearing loss. Noise Health 2014; 16: 257-264

[28] Gevrek F, Aydin D, Ozsoy S, Aygun H, Bicer C. Inhibition by Egb761 of the effect of cellphone radiation on the male reproductive system. Bratisl Lek Listy 2017; 118: 676-683

[29] Sharma M, Fitzpatrick AL, Arnold AM, Chi G, Lopez OL, Jenny NS, DeKosky ST. Inflammatory biomarkers and cognitive decline: the Ginkgo evaluation of memory study. J Am Geriatr Soc 2016; 64: 1171-1177

[30] Saini AS, Taliyan R, Sharma PL. Protective effect and mechanism of Ginkgo biloba extract-EGb 761 on STZ-induced diabetic cardiomyopathy in rats. Pharmacogn Mag 2014; 10: 172-178

[31] Wu JQ, Kosten TR, Zhang XY. Free radicals, antioxidant defense systems, and schizophrenia. Prog Neuropsychopharmacol Biol Psychiatry 2013; 46: 200-206

[32] Lee CY, Yang J], Lee SS, Chen C], Huang YC, Huang KH, Kuan YH. Protective effect of Ginkgo biloba leaves extract, EGb761, on endotoxin-induced acute lung injury via a JNK- and Akt-dependent NFkappaB pathway. J Agric Food Chem 2014; 62: 6337-6344
[33] Aziz TA, Hussain SA, Mahwi TO, Ahmed ZA. Efficacy and safety of Ginkgo biloba extract as an "add-on" treatment to metformin for patients with metabolic syndrome: a pilot clinical study. Ther Clin Risk Mang 2018; 14 ; 1219-1226

[34] Hirata BK, Banin RM, Dornellas AP, de Andrade IS, Zemdegs JC, Caperuto LC, Oyama LM, Ribeiro EB, Telles MM. Ginkgo biloba extract improves insulin signaling and attenuates inflammation in retroperitoneal adipose tissue depot of obese rats. Mediators Inflamm 2015; 2015: 419106

[35] Siegel G, Ermilov E, Knes O, Rodriguez M. Combined lowering of low grade systemic inflammation and insulin resistance in metabolic syndrome patients treated with Ginkgo biloba. Atherosclerosis 2014; 237: 584-588

[36] Fang H, Zhang C, Wang J, Xu Z, Qian C, Zhang L. Therapeutic effects of Ginkgo biloba extract against acute ischemic colitis. Medicine (Baltimore) 2018; 97: e12166

[37] Lebda MA, Sadek KM, Tohamy HG, Abouzed TK, Shukry M, Umezawa M El-Sayed YS. Potential role of alpha-lipoic acid and Ginkgo biloba against silver nanoparticles-induced neuronal apoptosis and blood-brain barrier impairments in rats. Life Sci 2018; 212: 251-260

[38] Zayed AE, Saleh A, Gomaa AMS, Abd-Elkareem M, Anwar MM, Hassanein KMA, Elsherbiny MM, Kotb AM. Protective effect of Ginkgo biloba and magnetized water on nephropathy in induced type 2 diabetes in rat. Oxid Med Cell Longev 2018; 2018: 1785614

[39] Rhee KJ, Lee CG, Kim SW, Gim DH, Kim HC, Jung BD. Extract of Ginkgo biloba ameliorates streptozotocin-induced type 1 diabetes mellitus and high-fat diet-induced type 2 diabetes mellitus in mice. Int J Med Sci 2015; 12: 987-994

[40] Ahmed HH, El-Abhar HS, Hassanin EAK, Abdelkader NF, Shalaby MB. Ginkgo biloba L. leaf extract offers multiple mechanisms in bridling Nmethylnitrosourea-mediated experimental colorectal cancer. Biomed Pharmacother 2017; 95: 387-393

[41] Liang Z, Bai S, Shen P, Hu Q, Wang X, Dong M, Wang W, Li ], Cheng K, Zhang S, Zou D, Han Y, Wang H, Xie P. GC-MS-based metabolomic study on the antidepressant-like effects of diterpene ginkgolides in mouse hippocampus. Behav Brain Res 2016; 314: 116-124

[42] Badem S, Ugurlucan M, El H, Sahin M, Uysal M, Sayin OA, Gurel B, Basaran M, Bayinder C, Alpagut U, Dayioglu E. Effects of Ginkgo biloba extract on spinal cord ischemia-reperfusion injury in rats. Ann Vas Surg 2014; 28: 1296-1305

[43] Trebaticka J, Durackova Z. Psychiatric disorders and polyphenols: can they be helpful in therapy? Oxid Med Cell Longev 2015; 2015: 248529

[44] Mi XS, Zhong JX, Chang RC, So KF. Research advances on the usage of traditional Chinese medicine for neuroprotection in glaucoma. J Integr Med 2013; 11: 233-240

[45] Zhang J, Wang J, Zhou GS, Tan YJ, Tao HJ, Chen JQ, Pu ZJ, Ma JY, She W, Kang A, Zhu Y, Liu P, Zhu ZH, Shi XQ, Tang YP, Duan JA. Studies of the anti-amnesic effects and mechanisms of single and combined use of donepezil and Ginkgo ketoester tablet on scopolamine-induced memory impairment in mice. Oxid Med Cell Longev 2019; 2019: 8636835

[46] Qin Y, Zhang Y, Tomic I, Hao W, Menger MD, Liu C, Fassbender K, Liu Y. Ginkgo biloba extract EGb 761 and its specific components elicit protective protein clearance through the autophagy-lysosomal pathway in tautransgenic mice and cultured neurons. J Alzheimers Dis 2018; 65: $243-$ 263

[47] El Tabaa MM, Sokkar SS, Ramadan ES, Abd El Salam IZ, Zaid A. Neuroprotective role of Ginkgo biloba against cognitive deficits associated with bisphenol A exposure: An animal model study. Neurochem Int 2017; 108: 199-212

[48] Ribeiro ML, Moreira LM, Arcari DP, Dos Santos LF, Marques AC, Pedrazzoli J jr., Cerutti SM. Protective effects of chronic treatment with a standardized extract of Ginkgo biloba I. in the prefrontal cortex and dorsal hippocampus of middle-aged rats. Behav Brain Res 2016; 313: 144-150 
[49] Zeng YQ, Wang Y], Zhou XF. Ginkgetin ameliorates neuropathological changes in app/ps1 transgenical mice model. J Prev Alzheimers Dis 2016; 3: 24-29

[50] Wan W, Zhang C, Danielsen M, Li Q, Chen W, Chan Y, Li Y. EGb761 improves cognitive function and regulates inflammatory responses in the APP/PS1 mouse. Exp Gerontol 2016; 81: 92-100

[51] Liu X, Hao W, Qin Y, Decker Y, Wang X, Burkart M, Schotz K, Menger MD, Fassbender K, Liu Y. Long-term treatment with Ginkgo biloba extract EGb 761 improves symptoms and pathology in a transgenic mouse model of Alzheimer's disease. Brain Behav Immun 2015; 46: 121-131

[52] Wang YQ, Wang MY, Fu XR, Peng Y, Gao GF, Fan YM, Duan XL, Zhao BL, Chang YZ, Shi ZH. Neuroprotective effects of ginkgetin against neuroinjury in Parkinson's disease model induced by MPTP via chelating iron. Free Radic Res 2015; 49: 1069-1080

[53] Harada S, Tsujita T, Ono A, Miyagi K, Mori T, Tokuyama S. Stachys sieboldii (Labiatae, Chorogi) protects against learning and memory dysfunction associated with ischemic brain injury. J Nutr Sci Vitaminol 2015; 61: $167-174$

[54] Liu Q, Jin Z, Xu Z, Yang H, Li L, Li G, Li F, Gu S, Zong S, Zhou J, Cao L, Wang Z, Xiao W. Antioxidant effects of ginkgolides and bilobalide against cerebral ischemia injury by activating the Akt/Nrf2 pathway in vitro and in vivo. Cell Stress Chaperones 2019; 24: 441-452

[55] Chen M, Zou W, Chen M, Cao L, Ding J, Xiao W, Hu G. Ginkgolide K promotes angiogenesis in a middle cerebral artery occlusion mouse model via activating JAK2/STAT3 pathway. Eur ] Pharmacol 2018; 833: 221-229

[56] Aydin D, Peker EG, Karakurt MD, Gurel A, Ayyildiz M, Cevher SC, Agar E, Dane S. Effects of Ginkgo biloba extract on brain oxidative condition after cisplatin exposure. Clin Invest Med 2016; 39: 27511

[57] Vaghef L, Bafandeh Gharamaleki H. Effects of physical activity and Ginkgo biloba on cognitive function and oxidative stress modulation in ischemic rats. Int J Angio 2017; 26: 158-164

[58] Yallapragada PR, Velaga MK. Effect of Ginkgo biloba extract on lead-induced oxidative stress in different regions of rat brain. J Environ Pathol Toxicol Oncol 2015; 34: 161-173

[59] Chen XJ, Ren SM, Dong JZ, Qiu CG, Chen YW, Tao HL. Ginkgo biloba extract-761 protects myocardium by regulating Akt/Nrf2 signal pathway. Drug Des Devel Ther 2019; 13: 647-655

[60] Wang Z, Zhang J, Ren T, Dong Z. Targeted metabolomic profiling of cardioprotective effect of Ginkgo biloba L. extract on myocardial ischemia in rats. Phytomedicine 2016; 23: 621-631

[61] Wu F, Shi W, Zhou G, Yao H, Xu C, Xiao W, Wu J, Wu X. Ginkgolide B functions as a determinant constituent of ginkgolides in alleviating lipopolysaccharide-induced lung injury. Biomed Pharmacother 2016; 81: 71-78

[62] Zhao Y, An X, Liu J, Liu S, Xu W, Yu X, Yu J. The improvement of oxidative stress by two proprietary herbal medicines in type 2 diabetes. Complement Ther Med 2018; 40: 120-125

[63] Jeong HS, Kim KH, Lee IS, Park JY, Kim Y, Kim KS, Jang HJ. Ginkgolide A ameliorates non-alcoholic fatty liver diseases on high fat diet mice. Biomed Pharmacother 2017; 88: 625-634

[64] Ye N, Wang H, Hong J, Zhang T, Lin C, Meng C. PXR mediated protection against liver inflammation by ginkgolide a in tetrachloromethane treated mice. Biomol There (Seoul) 2016; 24: 40-48
[65] Wang Y, Wang R, Wang Y, Peng R, Wu Y, Yuan Y. Ginkgo biloba extract mitigates liver fibrosis and apoptosis by regulating p38 MAPK, NF-kappaB/IkappaBalpha, and Bcl-2/Bax signaling. Drug Des Devel Ther 2015; 9: 6303-6317

[66] Li H, Qiu P, Wang J, Niu C, Pan S. Effects of compound Ginkgo biloba on intestinal permeability in rats with alcohol-induced liver injury. Food Funct 2015; 6: 470-478

[67] Ahmed Al, Lasheen NN, El-Zawahry KM. Ginkgo biloba ameliorates subfertility induced by testicular ischemia/reperfusion injury in adult wistar rats: a possible new mitochondrial mechanism. Oxid Med Cell Longev 2016; 2016: 6959274

[68] Mohamed NE, Abd El-Moneim AE. Ginkgo biloba extract alleviates oxidative stress and some neurotransmitters changes induced by aluminum chloride in rats. Nutrition 2017; 35: 93-99

[69] Lu Q, Hao M, Wu W, Zhang N, Isaac AT, Yin J, Zhu X, Du L, Yin X. Antidiabetic cataract effects of $\mathrm{GbE}$, rutin and quercetin are mediated by the inhibition of oxidative stress and polyol pathway. Acta Biochim Pol 2018; 65: $35-41$

[70] Sjostrand AP, Dogan R, Kocyigit A, Karatas E, Budak BB, Ozturan O. Therapeutic efficacy of Ginkgo biloba for early-period noise-induced hearing loss: an experimental animal study. Am J Otolaryngol 2016; 37: 416-424

[71] Kaur N, Dhiman M, Perez-Polo JR, Mantha AK. Ginkgolide B revamps neuroprotective role of apurinic/apyrimidinic endonuclease 1 and mitochondrial oxidative phosphorylation against Abeta25-35-induced neurotoxicity in human neuroblastoma cells. J Neurosci Res 2015; 93: 938947

[72] Lin H, Guo X, Zhang S, Dial SL, Guo L, Manjanatha MG, Moore MM, Mei N Mechanistic evaluation of Ginkgo biloba leaf extract-induced genotoxicity in L5178Y cells. Toxicol Sci 2014; 139: 338-349

[73] Parimoo HA, Sharma R, Patil RD, Sharma OP, Kumar P, Kumar N. Hepatoprotective effect of Ginkgo biloba leaf extract on lantadenes-induced hepatotoxicity in guinea pigs. Toxicon 2014; 81: 1-12

[74] Serrano-Garcia N, Pedraza-Chaverri ], Mares-Samano J], Orozco-Ibarra M Cruz-Salgado A, Jimenez-Anguiano A, Sotelo J, Trejo-Solis C. Antiapoptotic effects of EGb 761. Evid Based Complement Alternat Med 2013; 2013: 495703

[75] Choudhary S, Kumar P, Malik J. Plants and phytochemicals for Huntington's disease. Pharmacogn Rev 2013; 7: 81-91

[76] Wang J, Zhang L, Zhang Y, Luo M, Wu Q, Yu L, Chu H. Transcriptional upregulation centra of $\mathrm{HO}-1$ by EGB via the MAPKs/Nrf2 pathway in mouse C2C12 myoblasts. Toxicol In Vitro 2015; 29: 380-388

[77] Wang L, Bai Y, Wang B, Cui H, Wu H, Lv JR, Mei Y, Zhang JS, Liu S, Qi LW, Chen Y. Suppression of experimental abdominal aortic aneurysms in the mice by treatment with Ginkgo biloba extract (EGb 761). J Ethnopharmacol 2013; 150: 308-315

[78] Lu L, Wang S, Fu L, Liu D, Zhu Y, Xu A. Bilobalide protection of normal human melanocytes from hydrogen peroxide-induced oxidative damage via promotion of antioxidase expression and inhibition of endoplasmic reticulum stress. Clin Exp Dermatol 2016; 41: 64-73 